\title{
Random Walker Image Registration with Inverse Consistency
}

\author{
Lisa Y. W. Tang, Shawn Andrews, and Ghassan Hamarneh
}

\author{
Medical Image Analysis Lab, School of Computing Science, Simon Fraser University, Canada
}

\begin{abstract}
One important property of a registration solution is inverse consistency. While often overlooked, this property is critical in many medical applications, including radiationtherapy treatment planning and unbiased atlas-construction. In this paper, we propose a novel extension to the graphbased random walker image registration (RWIR) algorithm to ensure its inverse consistency. In contrast to many existing inverse-consistent algorithms, where two bi-directional transformations are independently sought and subsequently averaged, we calculate both transformations simultaneously by performing a constrained graph labeling in a common domain onto which both images are mapped, and employ a set of coupled labels so that both transformations are computed within a single optimization step. As our results on synthetic and real problems involving cardiac, thigh and brain images demonstrate, our method not only improved inverse consistency of RWIR, but also statistically significantly improved its accuracy. Comparison to another state-of-theart symmetric algorithm on various datasets also gave highly encouraging results.
\end{abstract}

\section{INTRODUCTION}

Random walker is a highly efficient optimizer [1]. Soon after its first adoption for stereo-correspondence [2], it was also deployed for medical image registration [1], leading to the random walker image registration algorithm (RWIR), which has since been evolving via various extensions that are either aimed at decreasing its computational complexity or improving its accuracy [3]-[8]. Empirical results reported in these extensions [3]-[8] all suggest that RWIR can be advanced to an accurate, efficient, and well-validated tool for practical real clinical applications.

In this paper, we further advance this promising registration framework by ensuring its inverse consistency, so that registration solutions remain consistent regardless of the order of the input images. While often overlooked, inverse consistency is an important criterion to consider, in addition to registration accuracy and other criteria like smoothness and invertibility of the solution [6], [8]. For instance, in image-guided and adaptive radiation therapy applications [9], where pre-therapy and daily CT images are co-registered, inverse consistent registrations will ensure that a pair of symmetric transformations can be obtained so that changes in radiation dose as captured by these images are invariant to changes in the reference frame. Along the same reasoning, inverse consistency is also critical in atlas-construction and atlas-based segmentation tasks. Even if an application does not explicitly require inverse consistency [10], imposing inverse consistency can significantly improve registration accuracy, as we will show in Section 3.

A common approach to ensuring inverse consistency is to take a bi-directional approach where two spatial transformations (for the forward and backward directions) are incrementally estimated [9]. This approach is adopted in many registration algorithms, including those proposed in recent literature [9], [11]-[14]. Performing two independent optimizations per iteration, however, at least requires twice as much resource as required by standard, inverse nonconsistent algorithms [9]. Furthermore, asymmetry of the registration energy is introduced when discretization and numerical approximations are performed within each coordinate frame independently [9]. All these factors suggest that alternative strategies are needed.

In this paper, rather than solving the forward and backward transformations independently, we solve both jointly in a single optimization, which stands in contrast to many recent state-of-the-art symmetric registration algorithms, e.g. [11]-[15]. We do this by operating in the common space onto which both images are mapped, and employ coupled displacement vectors to represent the updates. Through the common space where the data similarity is measured and spatial regularization is enforced, our method effectively avoids the aforementioned asymmetry. As we will show in Section 3, our extensions not only significantly improved the inverse consistency of RWIR, but also led to increased accuracy, using a decreased number of iterations.

We note that our approach of performing graph-labeling in a common space is most similar to the framework of [16]. However, this non-probabilistic framework uses the Primal-Dual optimization, which requires high memoryconsumption [11], and in order to decrease computational and memory costs, a sparse transformation representation, i.e. Free-Form Deformation (FFD), was used. Conversely, our probabilistic framework does not consume as much memory and thus can solve $\mathbf{U}$ non-parametrically, thereby allowing fine-scale deformations that can potentially lead to more accurate registrations. Moreover, to ensure invertibility, 
[16] restricted the maximum allowed displacement of the control points of the FFD to be no more than 0.4 times its grid-spacing. On the other hand, by adopting the framework of [6], our method ensures diffeomorphism without the need of imposing this constraint. In addition, via the RWIR formulation, our method inherently yields a probabilistic solution that allows for efficient uncertainty evaluation ${ }^{1}$, whereas uncertainty ("min-marginals") in the solutions of [16] must be computed via an independent step subsequent to optimization.

We also note that outside the discrete optimization paradigm for registration [9], there exist methods that do not perform independent optimizations to estimate the forward and backward transforms. However, as elaborated in [9], these methods either require solving a system of PDEs, which can be highly computationally intensive, or are based on assumptions that restrict incremental updates to be small (e.g. 0.4 voxels in [18]), thus leading to low "convergence rate" [9]. We bypass these issues by leveraging the global optimality and efficiency of the random walker solver.

\section{METHODS}

\subsection{Brief review of random walker image registration}

We aim to find a spatial transformation $T: \Omega \subset \mathbb{R}^{n} \mapsto \mathbb{R}^{n}$ that best aligns two images $I_{1}$ and $I_{2}$ of dimensionality $n$. The problem is generally cast as a search for a displacement vector field $\mathbf{U}, T(\mathbf{x})=\mathbf{x}+\mathbf{U}(\mathbf{x})$, that best minimizes an energy $E$ consisting of an image dissimilarity measure $\mathcal{D}$ and a spatial regularization term that measures the smoothness of U. In RWIR [1], the image is represented with a graph $\mathcal{G}(\mathcal{V}, \mathcal{E})$ where a pixel coordinate $\mathbf{x}_{p}$ is represented by a graph node $p \in \mathcal{V},|\mathcal{V}|=V$. Spatial connectivities between nodes are encoded by a Laplacian matrix $L$, which gives the effect of enforcing diffusion-based regularization on $\mathbf{U}$. A given sampling of the feasible solution space of $\mathbf{U}(\mathbf{x})$ is represented by a set of $K$ displacement labels $\mathcal{L}=\left\{\mathbf{v}_{k}\right\}$, $\mathbf{v}_{k} \in \mathbb{R}^{n}(k=1, \cdots, K)$. The image dissimilarity costs associated with $\mathcal{L}$ are then encoded by a $V \times K$ matrix $\mathbf{D}=\left(d_{p k}\right)$, whose elements may be computed as:

$$
d_{p k}=\exp \left(-\mathcal{D}\left(I_{1}\left(\mathbf{x}_{p}\right), I_{2}\left(\mathbf{x}_{p}+\mathbf{v}_{k}\right)\right),\right.
$$

such that low image dissimilarities between $I_{2}\left(\mathbf{x}_{p}+\mathbf{v}_{k}\right)$ and $I_{1}\left(\mathbf{x}_{p}\right)$ yield high likelihood values. The probabilistic field $\mathbf{P}: \Omega \mapsto \mathbf{s}\left(\mathbf{s} \in \Delta_{K}\right.$, the $K$-D unit simplex) is then computed by minimizing:

$E\left(\mathbf{P}_{k}\right)=\sum_{j=1, j \neq k}^{K} \mathbf{P}_{k}^{T} \Lambda_{j} \mathbf{P}_{k}+\left(1-\mathbf{P}_{k}\right)^{T} \Lambda_{k}\left(1-\mathbf{P}_{k}\right)+\alpha \mathbf{P}_{k}^{T} L \mathbf{P}_{k}$

where $\mathbf{P}_{k}$ denotes the $k$-th component of $\mathbf{P}$ and $\Lambda_{k}$ is a diagonal matrix with entries $\left[d_{1 k}, \cdots, d_{V k}\right]$. As [1], [3]

\footnotetext{
${ }^{1}$ Uncertainty information can be used, e.g., to infer registration error [4], and/or to improve registration performance [17].
}

present, the first two terms in (2) encourage $\mathbf{P}$ to be similar to $\mathbf{D}$, while the last term enforces diffusion regularization on $\mathbf{P}$. The solution $\mathbf{U}$ is then computed in closed-form by solving a linear system of equations. In [1], the sought displacement field $\mathbf{U}$ is then computed as the maximum $a$ posteriori solution, i.e. $\mathbf{U}(\mathbf{x})=\mathbf{v}_{k}$ whenever $\mathbf{P}_{p k}$ is the maximum probability of node $p$.

\subsection{Graph-labeling in the common space}

In the original RWIR framework [1], the solution is neither guaranteed to be symmetric nor diffeomorphic. Here, we extend [6] to impose both of these properties on the solution. Our algorithm adopts the standard multi-resolution approach where the solution is computed incrementally from a set of (update) displacement fields $\mathbf{V}^{1}$ and $\mathbf{V}^{2}$. However, rather than computing the update fields independently in each resolution level, we optimize the forward and backward update fields simultaneously. As mentioned in Section 1, this effectively reduces the computation by a half, which stands in contrast to many symmetric registration algorithms (e.g. [11]-[15]) that require two update calculations per iteration. Additionally, we employ random walker to compute globally optimal updates that can be arbitrarily large, subject to appropriate choice of $\mathcal{L}$ (i.e. the appropriate sampling resolution and size of the search region). Consequently, our method converges to the global optimum quickly, and as we will show in Section 3, it generally requires fewer iterations than the symmetric registration framework [15].

The key idea to facilitating the simultaneous estimation of the backward and forward updates is that we employ a label set of coupled displacement update vector pairs that would represent how the images will be mapped to a common space $\Omega^{c}$ from their respective domains, $\Omega^{1}$ and $\Omega^{2}$. We thus form a set of vector labels as $\mathcal{L}=\left\{\left(\mathbf{v}_{1},-\mathbf{v}_{1}\right), \cdots,\left(\mathbf{v}_{k},-\mathbf{v}_{k}\right)\right\}$, where $\mathbf{v}_{k} \in \mathbb{R}^{n}$ are uniformly sampled from the (semi hyperspherical) search space of the tangent vector space, such that the first (or second) element gives a mapping between $\Omega^{c}$ and $\Omega^{1}$ (or $\Omega^{2}$ ). Next, we calculate $D^{c}=\left(d_{p k}^{c}\right)$, the datalikelihood of assigning $\left(\mathbf{v}_{k},-\mathbf{v}_{k}\right)$ to node $\mathbf{x}_{p} \in \Omega^{c}$, as:

$$
\begin{aligned}
& d_{p k}^{c}= \\
& \exp \left(-\mathcal{D}\left(I_{1}\left(\mathbf{x}_{p}+\mathbf{V}^{1}\left(\mathbf{x}_{p}\right)+\mathbf{v}_{k}\right), I_{2}\left(\mathbf{x}_{p}+\mathbf{V}^{2}\left(\mathbf{x}_{p}\right)-\mathbf{v}_{k}\right)\right)\right. \\
& \exp \left(-\mathcal{D}\left(I_{2}\left(\mathbf{x}_{p}+\mathbf{V}^{2}\left(\mathbf{x}_{p}\right)-\mathbf{v}_{k}\right), I_{1}\left(\mathbf{x}_{p}+\mathbf{V}^{1}\left(\mathbf{x}_{p}\right)+\mathbf{v}_{k}\right)\right) .\right.
\end{aligned}
$$

Prior to solving (2), $\mathbf{D}^{c}$ is normalized to sum to unity. Note that $\mathbf{D}^{c}$ is symmetric, i.e. invariant to the order of the input images, and that spatial regularization is performed in $\Omega^{c}$, thus promoting symmetry in the problem formulation. Finally, to enforce diffeomorphism, we adopt the approach of [6] where we apply the exponential map to the optimized update fields [15]. 


\section{RESULTS}

\subsection{Synthetic experiments}

We apply synthetic warps to registered pairs of images to obtain groundtruth solutions. The warps were generated by randomly displacing control points of a free-form deformation model. The magnitude of the displacements were sampled from $\mathcal{N}(4,3)$ (in pixels). To evaluate registration performance, we quantified the registration error as the mean end-point-error (MEPE) between the recovered and the groundtruth warps. We also computed the inverse consistency error (ICE) of the solutions as $I C E=\frac{1}{2}\left(\left\|\mathrm{~T}^{f} \circ \mathrm{T}^{b}\right\|+\right.$ $\left.\left\|\mathrm{T}^{b} \circ \mathrm{T}^{f}\right\|\right)$. Based on subjective evaluation of a few initial trials, $\alpha$ was set to $0.025 \times V$ in all results reported.

Exp. 1) Proposed symmetric vs. non-symmetric RWIR [6]. We first examined how the proposed symmetric and diffeomorphic RWIR algorithm (denoted as SDRW) can improve inverse consistency and registration accuracy of the asymmetric version [6]. For this task, we performed a total of 150 synthetic trials. As test data, we randomly selected 50 pairs of aligned T1 and T2 images from the IXI brain MRI dataset ${ }^{2}$. Warps were then applied to one of the images to introduce misaligned pairs of multi-modal image pair. We then compared our SDRW with the (non-symmetric) diffeomorphic RWIR-based algorithm of [6] (denoted as DRW). Fig. 1a reports the MEPE and ICE averaged over these trials. Evidently, the ICE of the proposed SDRW is not only significantly lower than that of DRW, but also achieved higher registration accuracy. Similar results can be observed in Fig. 1b-c when we repeated the experiments over two pairs of thigh images from the Visible Human (VH) dataset ${ }^{3}$. Note that this was true regardless of the similarity measure employed, i.e. based on the MIND descriptor of [12] or normalized intensity gradients (NG).

Exp. 2) SDRW vs. non-graph based symmetric algorithm. We next compared the accuracy, inverse consistency, and convergence rate of our algorithm with an implementation of the symmetric diffeomorphic registration algorithm of [15], hereafter denoted as SLDemons. In this experiment, the same image pairs and similarity measure (squared intensity difference, SD) were used for all methods tested. Fig. 2 shows results from four sets of experiments, each with 60 trials of random warps applied to IXI brain MRI and cardiac MRI images at 4 different warp levels: $\mathcal{N}(2,3), \mathcal{N}(3,3)$, $\mathcal{N}(4,3), \mathcal{N}(5,3)$ (in pixels) for levels 1 to 4 , respectively. For all warp levels, the registration error of SDRW decreased at the fastest rate when compared to SLDemons [15] and DRW [6], especially for warp levels 3 and 4. Note also that our method gave lower ICE than that of DRW, regardless of warp levels. In addition, for specific choices of $\sigma$, SLDemons cannot ensure diffeomorphism (as similarly observed in [19]

\footnotetext{
${ }^{2}$ http://biomedic.doc.ic.ac.uk/

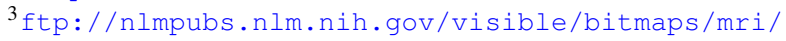

in the case of Diffeomorphic Demons) and yielded high ICE (e.g. Fig. $2 \mathrm{a} \sigma=0.5$ ).

\subsection{Registrations of brain MRI from a public dataset}

We next evaluated our method's performance on the CUMC12 public dataset containing 12 brain images, each with a 130-region segmentation. These fine-scale segmentations allow us to evaluate registration properly [20] and allow us to compare SDRW with a previous RWIR extension [3], as well as 15 other algorithms. For a fair comparison, we followed [3] in using the SD measure. A total of $12 \times 11$ pairwise registrations were performed. In summary, our method 1) achieved a Jaccard Index of 0.324, which was an improvement over the original framework [3] by 0.01 , and more importantly, 2) ensured inverse consistency in the solution, while [3] does not. We also note that our method achieved a target overlap of $0.485 \pm 0.041$, which, based on the figures reported in [14], ranks 6th out of the 17 methods compared. However, only 2 of the 5 more superior methods ensured symmetric solutions, and that these two these methods employ more advanced similarity measures, unlike the computationally more efficient SD measure that we have employed in this work.

\section{CONCLUSIONS}

This is the first work that extends RWIR to ensure its inverse consistency. We achieved this by performing probabilistic graph-labeling in the common space using a labelset of coupled pairs. Future research directions include using parallel computing to speed up all calculations and exploring the potential advantages of incorporating the method of [8] to enforce additional topology-preserving constraints on the update fields during optimization.

Acknowledgment. The authors are grateful to NSERC for partially funding this work.

\section{REFERENCES}

[1] D Cobzas and A Sen, "Random walks for deformable image registration," in MICCAI, 2011, pp. 557-565.

[2] R Shen, I Cheng, X Li, and A Basu, "Stereo matching using random walks," in $I C P R, 2008$.

[3] K Popuri, D Cobzas, and M Jagersand, "A variational formulation for discrete registration," in MICCAI, 2013, pp. 187-194.

[4] L Tang and G Hamarneh, "Random walks with efficient search and contextually adapted image similarity for deformable registration," in MICCAI 2013, 2013, vol. 8150, pp. 43-50.

[5] S Andrews, L Tang, and G Hamarneh, "Fast random walker image registration using precomputation," in $I S B I, 2014$, pp. $1-4$

[6] L Tang and G Hamarneh, "Random walker image registration with cost aggregation," in ISBI, 2014, pp. 1-4.

[7] S Andrews, L Tang, and G Hamarneh, "Fast random walker image registration using precomputation," in ISBI, 2013, pp. $1-8$. 
(a) IXI: PD+T2 (NGD)

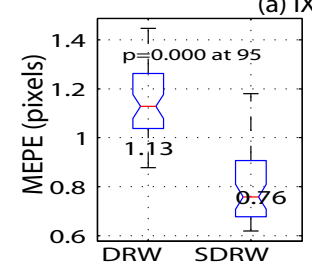

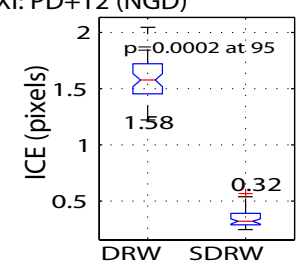

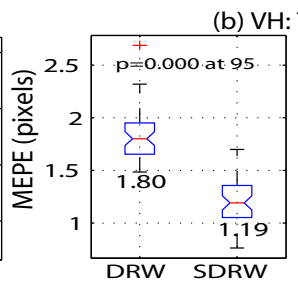

(b) $\mathrm{VH}: \mathrm{T} 2+\mathrm{T} 1$ (NGD)
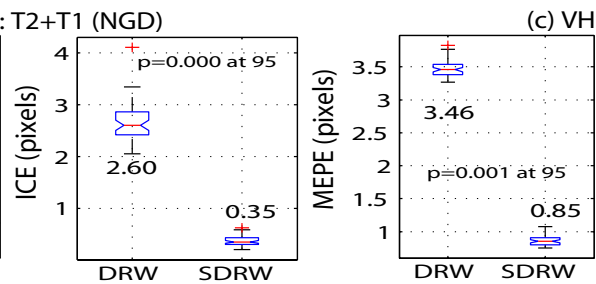

(c) VH: PD+T2 (MIND)

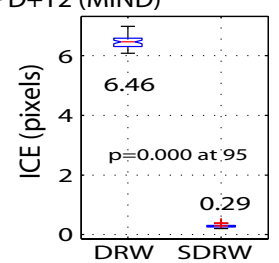

Fig. 1. Experiments ran on different multi-modal image pairs from the (a) IXI and (b-c) VH datasets, using 2 similarity measures. Evidently, SDRW gave MEPE and ICE that are statistically significantly lower than those of DRW.

(a) IXI Brain Pair 1 - level1

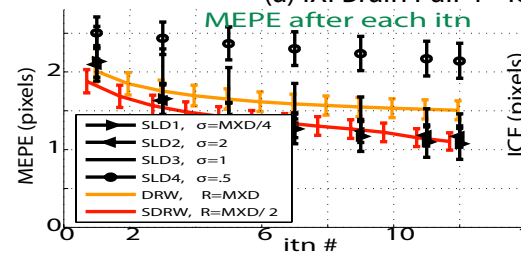

(c) IXI Brain Pair 3 - level3

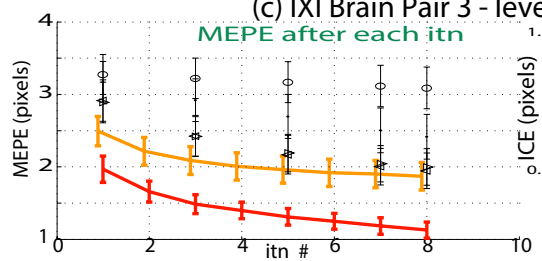

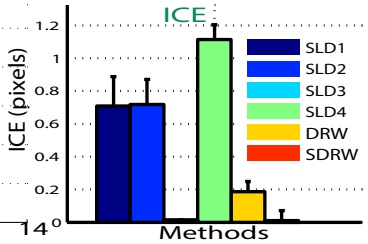

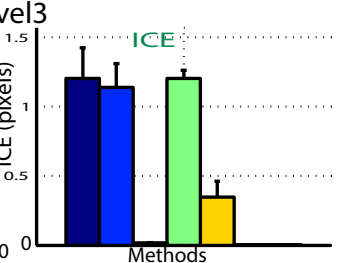

(b) IXI Brain Pair 2 - level2

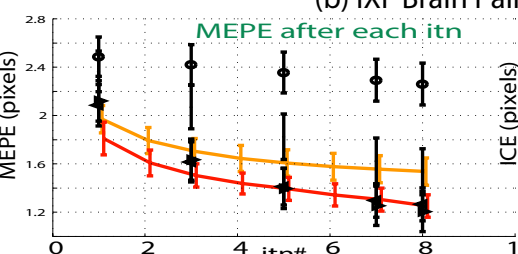

(d) Cardiac MRI - level4

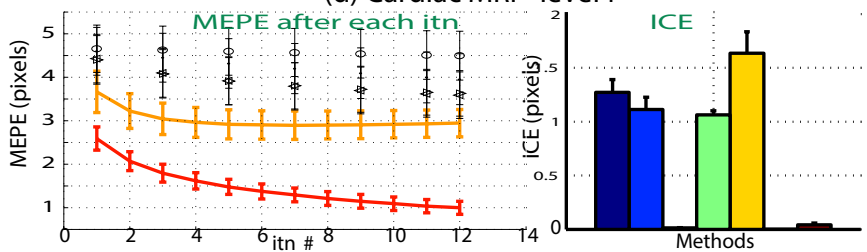

Fig. 2. Comparison to SLDemons [15] and DRW [6]. Plots showing reduction of MEPE and ICE over iteration for (a-c) 3 brain MRIs from the IXI dataset and a cardiac MRI. Evidently, the error decreased quickest when the proposed method (SDRW) was used. To avoid clutter, we have removed some data points for SLDemons (denoted with SLDx). Note how SLDemons was sensitive to parameter choice $\sigma$. For SDRW, we tested on two values of $\alpha$ on initial runs and found results to be similar. The sampling resolution of the search space defined by $R$ was simply set to MXD, which was set to the maximum displacement magnitude in each simulated warp.

[8] S Andrews, L Tang, and G Hamarneh, "Topology preservation and anatomical feasibility in random walker image registration," in MICCAI, 2014, pp. 210-217.

[9] A Sotiras, Discrete Image Registration : a Hybrid Paradigm, Phd thesis, Ecole Centrale Paris, Nov. 2011.

[10] M Sabuncu, T Yeo, K Van Leemput, T Vercauteren, and P Golland, "Asymmetric image-template registration," in MICCAI. 2009, pp. 565-573, Springer.

[11] M Heinrich, M Jenkinson, M Brady, and J Schnabel, "MRFbased deformable registration and ventilation estimation of lung CT," IEEE Transaction on Medical Imaging, vol. 32, no. 7, pp. 1239-48, 2013.

[12] M Heinrich, M Jenkinson, M Bhushan, T Matin, F Gleeson, S Brady, and J Schnabel, "MIND: Modality independent neighbourhood descriptor for multi-modal deformable registration," Medical Image Analysis, vol. 16, no. 7, 2012.

[13] H Lombaert and L Grady and X Pennec and A Nicolas and C Farida, "Spectral log-demons: Diffeomorphic image registration with very large deformations," IJCV, pp. 1-18, 2013.

[14] G Wu, M Kim, Q Wang, and D Shen, "S-HAMMER: Hierarchical attribute-guided, symmetric diffeomorphic registration for MR brain images," Human Brain Mapping, vol. 35, no. 3, 2014.

[15] T Vercauteren, X Pennec, A Perchant, and N Ayache, "Dif- feomorphic demons: Efficient non-parametric image registration," NeuroImage, vol. 45, pp. S61 - S72, 2009.

[16] A Sotiras and N Paragios, "Discrete symmetric image registration," in ISBI. 2012, IEEE.

[17] T Lotfi, L Tang, S Andrews, and G Hamarneh, "Improving probabilistic image registration via reinforcement learning and uncertainty evaluation.," in MLMI. 2013, vol. 8184 of Lecture Notes in Computer Science, pp. 187-194, Springer.

[18] D Yang, H Li, D.A. Low, J.O. Deasy, and I. El Naqa, "A fast inverse consistent deformable image registration method based on symmetric optical flow computation," in Signals, Systems and Computers, 2008 42nd Asilomar Conference on, Oct 2008, pp. 1931-1935.

[19] M Hernandez, S Olmos, and X Pennec, "Comparing algorithms for diffeomorphic registration: Stationary LDDMM and Diffeomorphic Demons," in MICCAI W'MFCA, 2008.

[20] T Rohlfing, "Image similarity and tissue overlaps as surrogates for image registration accuracy: Widely used but unreliable," Medical Imaging, IEEE Transactions on, vol. 31, no. 2, Feb 2012. 